\title{
SUPPLEMENT TO \\ BAYESIAN ESTIMATION OF SPARSE SIGNALS WITH A CONTINUOUS SPIKE-AND-SLAB PRIOR
}

\author{
By Veronika RočKOVÁ*, \\ University of Chicago ${ }^{\dagger}$
}

\section{Supplemental Proofs of Theorems.}

1.1. Proofs of Section 4. Throughout this section, we will be using the notation $\Delta^{L}$ and $\Delta^{U}$ introduced in (3.15). We also denote by

$$
\delta_{c}=\frac{1}{\lambda_{0}-\lambda_{1}} \log \left[\frac{1-\theta}{\theta} \frac{\lambda_{0}}{\lambda_{1}} \frac{c}{1-c}\right]
$$

and

$$
c_{+}=\frac{1}{2}\left(1+\sqrt{1-\frac{4}{\left(\lambda_{0}-\lambda_{1}\right)^{2}}}\right) \quad \text { and } \quad c_{-}=\frac{1}{2}\left(1-\sqrt{1-\frac{4}{\left(\lambda_{0}-\lambda_{1}\right)^{2}}}\right) .
$$

With this notation, $\delta_{c-}<\delta_{c+}$ are the two roots of the equation $\operatorname{pen}^{\prime \prime}(\beta)=1$.

1.1.1. Proof of Theorem 3.1. First, we assume that $L(\beta, y)$ has two modes. Denote by $\widehat{\beta}$ the global mode and by $\widetilde{\beta}$ the local mode. First, assume $\widehat{\beta}=0$ and $\left(|y|-\lambda_{1}\right)^{2}>2 \log \left[1 / p^{\star}(0)\right]$. By (3.10) we have

$$
y=\widetilde{\beta}+\operatorname{sign}(y) \lambda^{\star}(\widetilde{\beta}) .
$$

Because $\widetilde{\beta}$ is a local maximum, the second derivative test $\operatorname{pen}^{\prime \prime}(\widetilde{\beta})<1$ yields

$$
p^{\star}(\widetilde{\beta})\left[1-p^{\star}(\widetilde{\beta})\right]<1 /\left(\lambda_{0}-\lambda_{1}\right)^{2} .
$$

Because $\widetilde{\beta} \neq 0$, (1.4) implies that $|\widetilde{\beta}|>\delta_{c+}>0$, where $\delta_{c+}$ is defined in (3.14). Since $\widehat{\beta}=0$, we can write

$$
y^{2}<(y-\widetilde{\beta})^{2}+2 \log \left(\frac{\pi(0)}{\pi(\widetilde{\beta})}\right) .
$$

*Veronika.Rockova@Chicagobooth.edu. 
By noting

$$
\frac{\pi(0)}{\pi(\widetilde{\beta})}=\frac{p^{\star}(\widetilde{\beta})}{p^{\star}(0)} \exp \left(\lambda_{1}|\widetilde{\beta}|\right)
$$

and invoking (1.3), (1.5) is equivalent to

$$
y^{2}<\lambda^{\star}(\widetilde{\beta})^{2}+2 \lambda_{1}|y|-2 \lambda_{1} \lambda^{\star}(\widetilde{\beta})+2 \log \left(\frac{p^{\star}(\widetilde{\beta})}{p^{\star}(0)}\right) .
$$

Rearranging the terms above, we obtain

$$
\left(|y|-\lambda_{1}\right)^{2}<g(\widetilde{\beta})+2 \log \left[1 / p^{\star}(0)\right],
$$

where $g(\cdot)$ is defined in (3.13). We will need the following lemma.

Lemma 1.1. Assume $\left(\lambda_{0}-\lambda_{1}\right)>2$. The function $g(x)$ is symmetric and $g(|x|)$ is negative and monotone-increasing on $\left[\delta_{c+} ; \infty\right)$. Moreover, $\lim _{|x| \rightarrow \infty} g(x)=0$ and $g(|x|)$ has a minimum at $\delta_{c+}$ if $g(0)>0$.

Proof. Writing $g(x)=\left[1-p^{\star}(x)\right]^{2}\left(\lambda_{0}-\lambda_{1}\right)^{2}+2 \log p^{\star}(x)$, the condition $g(x)<0$ will be satisfied when

$$
\log p^{\star}(x)<-\frac{\left[1-p^{\star}(x)\right]^{2}}{2}\left(\lambda_{0}-\lambda_{1}\right)^{2} .
$$

Using $\log (x)<x-1$ for $x<1$, (1.8) will be satisfied when $p^{\star}(x)>1-$ $\frac{2}{\left(\lambda_{0}-\lambda_{1}\right)^{2}}$. Equating the derivative of $g(|x|)$ to zero yields

$$
2\left[1-p^{\star}(|x|)\right]\left(\lambda_{0}-\lambda_{1}\right)\left\{1-p^{\star}(|x|)\left[1-p^{\star}(|x|)\right]\left(\lambda_{0}-\lambda_{1}\right)^{2}\right\}=0
$$

We obtain the two solutions $\delta_{c-}<\delta_{c+}$ defined by (1.1) and (1.2). Because $|\widetilde{\beta}|>\delta_{c+}>0$ and $\operatorname{pen}^{\prime \prime}(|\widetilde{\beta}|)<1$, we have $g^{\prime}(|x|)>0$ on $\left[\delta_{c+}, \infty\right)$. Since $p^{\star}(x) \geq c_{+}>1-\frac{2}{\left(\lambda_{0}-\lambda_{1}\right)^{2}}$ on $\left[\delta_{c+}, \infty\right)$, we have $g(x)<0$ on $\left[\delta_{c+}, \infty\right)$. Because $g(|x|)$ is monotone increasing on $\left[\delta_{c+}, \infty\right), g(|x|)$ has a local minimum at $\delta_{c+}$. The value $g\left(\delta_{c+}\right)$ will be a global minimum if $g(0)>0$. Because $\lim _{|x| \rightarrow \infty} p^{\star}(x)=1$, we obtain $\lim _{|x| \rightarrow \infty} g(x)=0$.

According to Lemma 1.1 we have $g(x)<0$ for $|x| \geq \delta_{c}$. Because $|\widetilde{\beta}|>$ $\delta_{c}$, we have $g(\widetilde{\beta})<0$ and $(1.7)$ contradicts the assumption $\left(|y|-\lambda_{1}\right)^{2}>$ $2 \log \left[1 / p^{\star}(0)\right]$. Therefore $\widehat{\beta}$ has to be nonzero. Next, assume $\left(|y|-\lambda_{1}\right)^{2}<$ $2 \log \left[1 / p^{\star}(0)\right]-d$. Since $-d=g\left(\delta_{c+}\right)$ is the minimum of the function $g(x)$, we obtain (1.7), which is equivalent to $\widehat{\beta}=0$. The selection threshold can be 
written as $\Delta=\sqrt{2 \log \left[1 / p^{\star}(0)\right]+g(\widetilde{\beta})}+\lambda_{1}$. Note that $|\widetilde{\beta}|>\delta_{c+}$. By Lemma 1.1, $g(x)$ is negative and monotone increasing on $\left[\delta_{c+} ; \infty\right)$ with a minimum at $\delta_{c+}$, we have $\Delta^{L}<\Delta \leq \Delta^{U}$.

Now, assume that the posterior is unimodal. First, let $\widehat{\beta} \neq 0$ and $|y|<\Delta$. Then $|y|>\lambda^{\star}(0)$ and because $g(0)>0$ we have $\lambda^{\star}(0)>\Delta$, which yields a contradiction. Next, assume $\widehat{\beta}=0$ and $|y|>\Delta$. Denote by $x_{0} \in\left(0, \delta_{c+}\right)$ the root of $g(x)=0$. Then, it is necessary that $|y|<\lambda^{\star}\left(x_{0}\right)+x_{0}$. We show that $\lambda^{\star}\left(x_{0}\right)+x_{0}<\Delta$, yielding a contradiction. Because $g\left(x_{0}\right)=0$, we have $\lambda^{\star}\left(x_{0}\right)+x_{0}=\lambda_{1}+\sqrt{2 \log \left[1 / p^{\star}\left(x_{0}\right)\right]}+x_{0}$. Define $h(x)=x+\sqrt{2 \log \left[1 / p^{\star}(x)\right]}$, then $h^{\prime}\left(x_{0}\right)=0$ and $h^{\prime}(x)<0$ for $x \in\left(0, x_{0}\right)$. Therefore $h\left(x_{0}\right)<h(0)$ and $\lambda^{\star}\left(x_{0}\right)+x_{0}<\Delta$.

Lemma 1.2. Let $d=-g\left(\delta_{c+}\right)$. Then $0<d<2-\left(\frac{1}{\lambda_{0}-\lambda_{1}}-\sqrt{2}\right)^{2}$.

Proof. Because $p^{\star}\left(\delta_{c+}\right)=c_{+}=\left(1-c_{-}\right)$. We can invoke the inequality $1-\sqrt{1-x^{2}}>x^{2} / 2$ for $0<x<1$ to obtain $\left(\lambda_{0}-\lambda_{1}\right)^{2}\left(1-c_{+}\right)^{2}>1 /\left(\lambda_{0}-\lambda_{1}\right)^{2}$. Because $c_{+}>1-\frac{2}{\left(\lambda_{0}-\lambda_{1}\right)^{2}}>0.5$ and $\log \left(1-x^{2}\right)>-x$ for $0<x<0.5$, we have $\log c_{+}>-\frac{\sqrt{2}}{\lambda_{0}-\lambda_{1}}$. Altogether, we have

$$
g\left(\delta_{c+}\right)>\frac{1}{\left(\lambda_{0}-\lambda_{1}\right)^{2}}-\frac{\sqrt{2}}{\lambda_{0}-\lambda_{1}}=\left(\frac{1}{\lambda_{0}-\lambda_{1}}-\sqrt{2}\right)^{2}-2 .
$$

Lemma 1.1 yields $f\left(\delta_{c+}\right)<0$, which completes the proof.

\subsection{Proofs of Section 4.}

1.2.1. Proof of Theorem 4.1. The risk of the Spike-and-Slab LASSO estimator $\widehat{\boldsymbol{\beta}}(3.7)$ can be written as

$$
R(\widehat{\boldsymbol{\beta}})=\sum_{i=1}^{p_{n}} \mathrm{E}_{\beta_{0 i}}\left[\widehat{\beta}_{i}-\beta_{0 i}\right]^{2}+\sum_{i=p_{n}+1}^{n} \mathrm{E}_{0}\left[\widehat{\beta}_{i}^{2}\right],
$$

where $p_{n}=\left\|\boldsymbol{\beta}_{0}\right\|_{0}$. We begin by finding an upper bound to the first summand in (1.9). We have

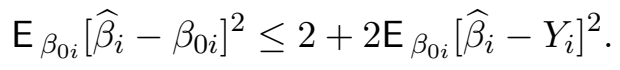

By Theorem 3.1 and because $\left|\widehat{\beta}_{i}\right|<\left|Y_{i}\right|$, we can write

$$
\mathrm{E}_{\beta_{0 i}}\left[\widehat{\beta}_{i}-Y_{i}\right]^{2} \leq \mathrm{E}_{\beta_{0 i}}\left[Y_{i}^{2} \mathbb{I}\left(\left|Y_{i}\right| \leq \Delta\right)\right]+\mathrm{E}_{\beta_{0 i}}\left[\lambda^{\star}\left(\widehat{\beta}_{i}\right)^{2} \mathbb{I}\left(\left|Y_{i}\right|>\Delta\right)\right] .
$$


Because $\left|\widehat{\beta}_{i}\right|>\delta_{c+}$, we have $p^{\star}\left(\widehat{\beta}_{i}\right)>c_{+}$when $\left|Y_{i}\right|>\Delta$, where $c_{+}$is defined in the proof of Lemma 3.1. Then, we have $\lambda^{\star}\left(\widehat{\beta}_{i}\right)<c_{+}\left(\lambda_{1}-\lambda_{0}\right)+\lambda_{0}$ when $\left|Y_{i}\right|>\Delta$. Next, we obtain

$$
\mathrm{E}_{\beta_{0 i}}\left[\widehat{\beta}_{i}-Y_{i}\right]^{2} \leq \Delta^{2}+\left[\lambda_{1}+\left(1-c_{+}\right)\left(\lambda_{0}-\lambda_{1}\right)\right]^{2} .
$$

Because $c_{+}\left(1-c_{+}\right)=1 /\left(\lambda_{0}-\lambda_{1}\right)^{2}$ and $c_{+}>0.5$, we have $\left(1-c_{+}\right)\left(\lambda_{0}-\lambda_{1}\right)<$ $2 /\left(\lambda_{0}-\lambda_{1}\right)$. Since $\Delta^{2}<4 \log \left[1 / p^{\star}(0)\right]+2 \lambda_{1}^{2}$ and because $\lambda_{1}<\mathrm{e}^{-2}<1$ and $\lambda_{0}-\lambda_{1}>2$, then

$$
\mathrm{E}_{\beta_{0 i}}\left[\widehat{\beta}_{i}-Y_{i}\right]^{2} \leq 4 \log \left[1 / p^{\star}(0)\right]+2 .
$$

Together with (1.10), this yields

$$
\mathrm{E}_{\beta_{0 i}}\left[\widehat{\beta}_{i}-\beta_{0 i}\right]^{2} \leq 8\left[\log \left[1 / p^{\star}(0)\right]+1\right] .
$$

Now we continue with the second summand in (1.9). Denote by $\phi(x)$ the standard normal density. Because $\left|\widehat{\beta}_{i}\right|<\left|Y_{i}\right|$ and $\Delta^{L}<\Delta \leq \Delta^{U}$, we can write

$$
\mathrm{E}_{0}\left[\widehat{\beta}_{i}^{2}\right]<\mathrm{E}_{0}\left[Y_{i}^{2} \mathbb{I}\left(\left|Y_{i}\right|>\Delta\right)\right]<\mathrm{E}_{0}\left[Y_{i}^{2} \mathbb{I}\left(\left|Y_{i}\right|>\Delta^{L}\right)\right]<4 \Delta^{L} \phi\left(\Delta^{L}\right),
$$

where the last inequality uses Mills's ratio (as in [?]). Because $\Delta^{L}>$ $\sqrt{2 \log \left[1 / p^{\star}(0)\right]-d}$, we have $\phi\left(\Delta^{L}\right)<1 / \sqrt{2 \pi} \mathrm{e}^{d / 2} p^{\star}(0)$. Because $\Delta^{L}<\sqrt{2 \log \left[1 / p^{\star}(0)\right]}+$ $\lambda_{1}$ and $\lambda_{1}<\mathrm{e}^{-2}<\sqrt{2}$, (1.11) can be further simplified as

$$
\mathrm{E}_{0}\left[\widehat{\beta}_{i}^{2}\right]<\frac{4 \mathrm{e}^{d / 2}}{\sqrt{\pi}} p^{\star}(0)\left[\sqrt{\log \left[1 / p^{\star}(0)\right]}+1\right]
$$

Altogether, we obtain

$(1.12)$

$\mathrm{E}_{\boldsymbol{\beta}_{0}}\left\|\boldsymbol{\beta}-\boldsymbol{\beta}_{0}\right\|^{2}<8 p_{n}\left(1+\log \left[1 / p^{\star}(0)\right]\right)+4 / \sqrt{\pi}\left(n-p_{n}\right) \mathrm{e}^{d} p^{\star}(0)\left(1+\sqrt{\log \left[1 / p^{\star}(0)\right]}\right)$.

\subsubsection{Proof of Theorem 4.2.}

Proof. Without loss of generality, we will assume $\frac{1-\theta}{\theta} \frac{\lambda_{0}}{\lambda_{1}}=n^{\alpha+\nu} / \lambda_{1}$. Denote by $Q(\boldsymbol{\beta})=-\frac{1}{2}\left\|\boldsymbol{y}^{(n)}-\boldsymbol{\beta}\right\|^{2}+\log \pi(\boldsymbol{\beta} \mid \theta)$, where $\pi(\boldsymbol{\beta} \mid \theta)$ is the SSL prior arising from (2.1) and (2.2). Using the global optimality $0 \geq Q\left(\boldsymbol{\beta}_{0}\right)-Q(\widehat{\boldsymbol{\beta}})$, we can write

$$
0 \geq\left\|\widehat{\boldsymbol{\beta}}-\boldsymbol{\beta}_{0}\right\|^{2}-2 \varepsilon^{\prime}\left(\widehat{\boldsymbol{\beta}}-\boldsymbol{\beta}_{0}\right)+2 \log \left(\frac{\pi\left(\boldsymbol{\beta}_{0} \mid \theta\right)}{\pi(\widehat{\boldsymbol{\beta}} \mid \theta)}\right) .
$$


Now, we focus on a set $\tau_{0}=\left\{\boldsymbol{y}^{(n)}:\left\|\boldsymbol{y}^{(n)}-\boldsymbol{\beta}_{0}\right\|_{\infty} \leq \bar{\lambda}\right\}$, where $\bar{\lambda}=2 \sqrt{\log n}$. This set has a large probability under the generative model, i.e. $\mathrm{P}\left(\tau_{0}\right) \geq$ $1-\frac{2}{n}\left[\right.$ ? ] . Conditioning on the set $\tau_{0}$, we use the Hölder inequality $\left|\boldsymbol{\alpha}^{\prime} \boldsymbol{\beta}\right| \leq$ $|\boldsymbol{\alpha}|_{\infty}|| \boldsymbol{\beta} \|_{1}$ to find that

$$
0 \geq\left\|\widehat{\boldsymbol{\beta}}-\boldsymbol{\beta}_{0}\right\|^{2}-2 \bar{\lambda}\left\|\widehat{\boldsymbol{\beta}}-\boldsymbol{\beta}_{0}\right\|_{1}+2 \log \left(\frac{\pi\left(\boldsymbol{\beta}_{0} \mid \theta\right)}{\pi(\widehat{\boldsymbol{\beta}} \mid \theta)}\right) .
$$

Denote by $\boldsymbol{\Delta} \equiv \widehat{\boldsymbol{\beta}}-\boldsymbol{\beta}_{0}$. Using the fact $\|\boldsymbol{\Delta}\|_{1} \leq\|\boldsymbol{\Delta}\|\|\boldsymbol{\Delta}\|_{0}^{1 / 2}$, we have

$$
0 \geq\|\boldsymbol{\Delta}\|^{2}-2 \bar{\lambda}\|\boldsymbol{\Delta}\|\|\boldsymbol{\Delta}\|_{0}^{1 / 2}+2 \log \left(\frac{\pi\left(\boldsymbol{\beta}_{0} \mid \theta\right)}{\pi(\widehat{\boldsymbol{\beta}} \mid \theta)}\right) .
$$

Because $p^{\star}\left(\widehat{\beta}_{j}\right)>c_{+}$whenever $\widehat{\beta}_{j} \neq 0$, we can write

$$
\begin{aligned}
\log \left[\frac{\pi\left(\boldsymbol{\beta}_{0} \mid \theta\right)}{\pi(\widehat{\boldsymbol{\beta}} \mid \theta)}\right] & \geq-\lambda_{1}\left\|\boldsymbol{\beta}_{0}-\widehat{\boldsymbol{\beta}}\right\|_{1}+\sum_{j=1}^{n} \log \left[\frac{p^{\star}\left(\widehat{\beta}_{j}\right)}{p^{\star}\left(\beta_{0 j}\right)}\right] \\
& \geq-\lambda_{1}\left\|\boldsymbol{\beta}_{0}-\widehat{\boldsymbol{\beta}}\right\|_{1}+\widehat{p}_{n} b+\left(\widehat{p}_{n}-p_{n}\right) \log \left[1 / p^{\star}(0)\right],
\end{aligned}
$$

where $0>b \equiv \log c_{+}>\log 0.5$ is a constant very close to 0 and where $\lim _{\lambda_{0} \rightarrow \infty} b=0$. To continue with (1.15), we can write $(1.16)$

$$
\left[\|\boldsymbol{\Delta}\|-\left(\bar{\lambda}+\lambda_{1}\right)\|\boldsymbol{\Delta}\|_{0}^{1 / 2}\right]^{2}+2\left(\widehat{p}_{n}-p_{n}\right) \log \left[1 / p^{\star}(0)\right] \leq\left(\bar{\lambda}+\lambda_{1}\right)^{2}\|\boldsymbol{\Delta}\|_{0}-2 \widehat{p}_{n} b .
$$

With (1.16) and using the fact $\|\boldsymbol{\Delta}\|_{0} \leq \widehat{p}_{n}+p_{n}$, we obtain

$$
2\left(\widehat{p}_{n}-p_{n}\right) \log \left[1 / p^{\star}(0)\right] \leq\left(\bar{\lambda}+\lambda_{1}\right)^{2}\left(\widehat{p}_{n}+p_{n}\right)-2 \widehat{p}_{n} b
$$

which is equivalent to writing

$$
\widehat{p}_{n} \leq p_{n}(1+C),
$$

where

$$
C=\frac{2\left(\bar{\lambda}+\lambda_{1}\right)^{2}}{2 \log \left[1 / p^{\star}(0)\right]-\left(\bar{\lambda}+\lambda_{1}\right)^{2}+2 b}
$$

and where $C=\mathcal{O}(1)$ under the given choice of hyper-parameters. Moreover, with $\lambda_{1}<\mathrm{e}^{-1}$ and $\frac{1-\theta}{\theta} \frac{\lambda_{0}}{\lambda_{1}}=n^{\alpha+\nu} / \lambda_{1}$, we have $2 \log \left[1 / p^{\star}(0)\right]-2\left(\bar{\lambda}+\lambda_{1}\right)^{2}+$ $2 b>2\left(\alpha+\nu-4-4 \lambda_{1}\right) \log n+2 \log \left(1 / \lambda_{1}\right)-2 \lambda_{1}^{2}+2 b>0$ for some suitable $c>0$. This inequality yields $0<C<2$. 
What remains to be shown is that $p_{n} \leq \widehat{p}$ under the suitable beta-min condition. We prove this statement by contradiction. Assume $\widehat{p}_{n}<p_{n}$ and denote by $q_{n}=p_{n}-\widehat{p}_{n}>0$. We continue with (1.15) and write

$$
0 \geq\|\boldsymbol{\Delta}\|\left[\|\boldsymbol{\Delta}\|-2\left(\bar{\lambda}+\lambda_{1}\right)\|\boldsymbol{\Delta}\|_{0}^{1 / 2}\right]-2 q_{n} \log \left[1 / p^{\star}(0)\right]+2 \widehat{p}_{n} b .
$$

Because $\|\boldsymbol{\Delta}\|_{0}<(1+C) p_{n}$, we can write

$0 \geq\|\boldsymbol{\Delta}\|\left[\|\boldsymbol{\Delta}\|-2\left(\bar{\lambda}+\lambda_{1}\right) \sqrt{(1+C) p_{n}}\right]-2 q_{n} \log \left[1 / p^{\star}(0)\right]+2 \widehat{p}_{n} \log 0.5$.

Assuming the minimal-strength condition $\left|\beta_{0 i}\right|>b_{0} \geq D \sqrt{p_{n} \log n}$ when $\beta_{0 i} \neq 0$, we can write

$$
\frac{1}{2}\|\boldsymbol{\Delta}\|>\frac{\sqrt{q_{n}}}{2} b_{0} \geq \frac{D}{2} \sqrt{q_{n} p_{n} \log n}>2\left(\bar{\lambda}+\lambda_{1}\right) \sqrt{p_{n}(1+C)}
$$

for a suitably large $D>0$. Assuming $\frac{1-\theta}{\theta} \frac{\lambda_{0}}{\lambda_{1}}=n^{\alpha+\nu} / \lambda_{1},(1.17)$ yields a contradiction

$$
\begin{aligned}
0 & \geq \frac{1}{2}\|\boldsymbol{\Delta}\|^{2}-2 q_{n} \log \left(1+n^{\alpha+\nu} / \lambda_{1}\right)+2 \widehat{p}_{n} \log 0.5 \\
& >\frac{D^{2}}{2} q_{n} p_{n} \log n-2 q_{n} \log \left(1+n^{\alpha+\nu} / \lambda_{1}\right)+2 \widehat{p}_{n} \log 0.5>0
\end{aligned}
$$

for $D$ sufficiently large.

\author{
VERONIKA RoČKOVÁ \\ BoOth SCHOOL OF Business \\ UNIVERSITY OF CHICAGO \\ 369 Carles M. Harper Center \\ 5807 S. Woodlawn Avenue \\ Chicago, IL 60637
}

